\title{
Local active control of road noise inside a vehicle
}

\author{
Woomin Jung*, Stephen J. Elliott, Jordan Cheer \\ Institute of Sound and Vibration Research, \\ University of Southampton, SO17 1BJ, United Kingdom
}

\begin{abstract}
The performance is investigated of a multichannel feedforward headrest system for the active control of interior road noise around a listener's ears in a vehicle cabin. An active headrest system is combined with the remote microphone technique so that direct measurement of the error signals at the listener's ears are not necessary. The formulation of the optimal observation filter for the remote microphone technique and the optimal controller for active control is presented in the time domain, to sense and control broadband random road noise. The estimation accuracy of the remote microphone technique is investigated through simulations, using the signals measured at a number of monitoring microphones and error microphones in the cabin of a large SUV. It is shown that disturbances at the virtual error microphones can be accurately estimated by a causal observation filter. The attenuation performance of the active headrest system using the remote microphone technique is also predicted offline, using measured plant responses, disturbance signals and reference signals, which shows that several peaks in the spectrum of the road noise can be reduced at frequencies of up to $1 \mathrm{kHz}$. The effects of delays and head movements on the attenuation performance are also studied.
\end{abstract}

Keywords: Active sound control, Local active noise control, Active headrest system, Remote microphone technique, Road noise, Vehicle interior noise

\section{Introduction}

Interior noise in road vehicles can be mainly categorised as either powertrain noise, tyre-road noise or aerodynamic noise. When a vehicle is driving at a relatively low speed, so that it does not produce a significant level of aerodynamic noise, the front structure-borne, rear structure-borne and powertrain air-

5 borne noise are the primary noise sources below $500 \mathrm{~Hz}$ [1]. The front structure-borne noise is generally produced from vibrations of the engine powertrain and tyre-road interaction and the rear structure-borne noise is mainly due to the tyre-road interaction. In the $500 \mathrm{~Hz}$ to $1,000 \mathrm{~Hz}$ frequency range, the tyre-road air-borne noise increases and becomes one of the primary noise sources. At this frequency range, although the front structure-borne noise and powertrain air-borne noise decrease, the rear structure-borne noise is

10 still generally the dominant noise source $[2,3]$. Therefore, noise from the interaction between the tyre and road is a main concern when attempting to control the interior noise in the frequency range up to 1,000 Hz. In particular, the influence of tyre-road noise on the interior noise is increasingly important because noise from the powertrain is continuously being reduced, by either improved powertrain design techniques or the application of the electric motor as an alternative to the internal combustion engine. Therefore, luxury 15 car manufactures are currently becoming concerned about mid-frequency road noise that may not be easily controlled passively.

\footnotetext{
*Corresponding author

Email addresses: wj3e13@soton.ac.uk (Woomin Jung), S.J.Elliott@soton.ac.uk (Stephen J. Elliott), J.Cheer@soton.ac.uk (Jordan Cheer)
} 
Active sound control in vehicles has been widely studied $[4,5,6]$. Although passive methods have been widely used to control NVH (Noise, Vibration and Harshness) in the vehicle cabin, research and development of electronic devices such as DSP (Digital Signal Processor), sensors and actuators may allow cost-effective

20 and practical implementations of active sound control [7,8]. Research into the active control of sound for vehicle interior noise is mainly concerned with two areas: powertrain noise control and road-tyre induced noise control.

Powertrain noise is mainly tonal and recently several systems for the active control of the low frequency components of this noise have been implemented by manufacturers $[9,10]$. The active control of the random 25 road noise in vehicles has also been investigated $[6,8,11,12]$, but the performance is also limited to low frequencies, typically below $300 \mathrm{~Hz}$ if the loudspeakers and microphones used for control are remote from the listener's head. Control of higher-frequency road noise may be possible with local control systems, such as those using loudspeakers and microphones in the headrests $[13,14,15]$. The proximity between the secondary loudspeakers and the error sensors in a local active headrest system can provide several advantages over a

30 global active control system, including low noise enhancement at other positions, short acoustic propagation delays in the control path and simpler plant responses between the secondary loudspeakers and the error sensors.

Despite these advantages, the direct installation of the error sensors in the desired control positions has limited the practical application of the active headrest system. For example, passengers in a car are unlikely

35 to accept the need to wear in-ear microphones over long periods of time for local active control. Although a number of virtual sensing algorithms have been proposed to avoid the use of such in-ear microphones $[16$, $17,18]$, the zone of quiet around the virtual error sensor, within which more than $10 \mathrm{~dB}$ attenuation is achieved, is limited to a diameter of about 1/10 of an acoustic wavelength in a pure tone diffuse field, which is about $3.4 \mathrm{~cm}$ at $1 \mathrm{kHz}$ [19]. Therefore, any separation between the virtual error sensors and the 40 targeted control regions can lead to the zone of quiet being outside of the desired region of control, and noise enhancement may even be generated due to constructive interference between the disturbance and control signals. To overcome this limitation, tracking of the head position and repositioning of the zone of quiet has been suggested by a number of authors [20, 21, 22]. The effect of the remote microphone technique and the head-tracking on the practical application of local active noise control was the motivation for this paper to

45 improve the controllable frequency range and the attenuation performance of the active headrest system in the automotive road noise control problem.

The aim of this paper is to investigate the performance of such a local control algorithm using direct measurements of the pressures and acoustic responses in a vehicle. The nearfield estimation accuracy of the remote microphone technique is initially considered when the causal optimal observation filter is designed

50 from measurements of the interior pressure at a number of monitoring microphones around a car cabin and two error microphones at the ears of a dummy head. To improve the causality of the remote microphone technique, this paper uses an improved remote microphone technique using additional delays, as suggested in [23]. It is then shown that a multichannel active headrest system with a causal controller can be combined with the remote microphone technique to reduce broadband interior road noise. The effect of head-tracking 55 on the nearfield estimation and the attenuation performance is also investigated, when the dummy head is located at different positions.

The formulation of such a control strategy in the time domain is considered first, in Section 2 , and measured signals from microphones positioned in the rear right seat of a large SUV are then used in Section 3 to estimate the performance of the remote microphone technique in practice. Section 4 presents the

60 attenuation performance of the active headrest system combined with the remote microphone technique to reduce broadband random road noise. Finally, Section 5 presents the conclusions of this work.

\section{Formulations for active control combined with the remote microphone technique}

\subsection{Optimal feedforward active control using the standard remote microphone technique}

To estimate virtual error signals at desired positions using signals measured at the remote monitoring 65 microphones and reduce disturbance signals at the virtual error sensors, the remote microphone technique 


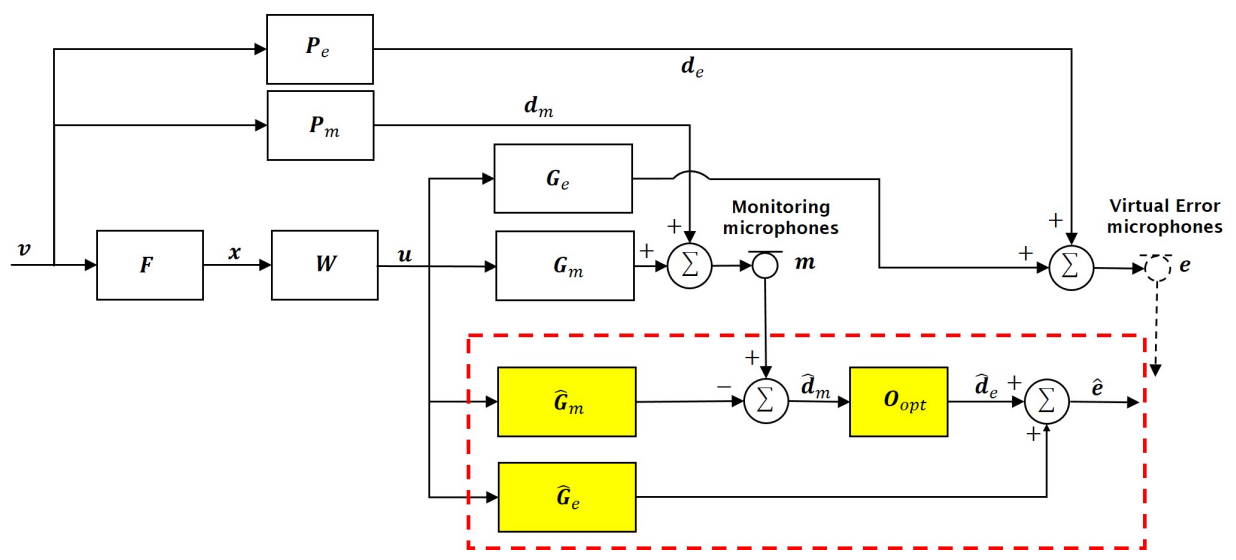

Figure 1: Block diagram of the feedforward active control algorithm, combined with the standard remote microphone technique in a dashed rectangle.

is combined with a feedforward active control system and the block diagram of the combined algorithm is shown in Fig. 1 [20, 24]. For stationary random broadband noise, it is required that the observation filter and the controller for active sound control are causally constrained. Therefore, we will investigate the nearfield estimation and attenuation performance in the time domain.

70 In Fig. 1, $N_{v}$ primary sources, which have a vector of source signals at the $n$th sample time, $\boldsymbol{v}(n)=$ $\left[v_{1}(n), v_{2}(n) \ldots v_{N_{v}}(n)\right]^{\mathrm{T}}$, are assumed to produce a vector of $N_{e}$ disturbance signals, $\boldsymbol{d}_{e}(n)=\left[d_{e 1}(n), d_{e 2}(n) \ldots d_{e N_{e}}(n)\right]^{\mathrm{T}}$ at the virtual error microphones and a vector of $N_{x}$ reference sensor signals, $\boldsymbol{x}(n)=\left[x_{1}(n), x_{2}(n) \ldots x_{N_{x}}(n)\right]^{\mathrm{T}}$ via matrices of transfer responses, $\mathbf{P}_{e}$ and $\mathbf{F}$, respectively. A vector of control signals for the $N_{u}$ secondary sources, $\boldsymbol{u}(n)=\left[u_{1}(n), u_{2}(n) \ldots u_{N_{u}}(n)\right]^{\mathrm{T}}$, is produced and the $n_{u}$ th control signal, $u_{n_{u}}(n)$, is obtained

75 from the sum of the contributions from the $N_{x}$ reference sensor signals, each filtered by an Ith order FIR control filter with coefficients $w_{n_{u} n_{x} i}$, so that

$$
u_{n_{u}}(n)=\sum_{n_{x}=1}^{N_{x}} \sum_{i=0}^{I-1} w_{n_{u} n_{x} i} x_{n_{x}}(n-i) .
$$

The control signals are transmitted to the virtual error microphones, via a matrix of plant responses, $\mathbf{G}_{e}$ to minimise the disturbance signals, $\boldsymbol{d}_{e}(n)$, and therefore, signals at the $n_{e}$ th virtual error microphone, $e_{n_{e}}(n)$ can be written as [25]

$$
e_{n_{e}}(n)=d_{e n_{e}}+\sum_{n_{u}=1}^{N_{u}} \sum_{k=0}^{K-1} \sum_{n_{x}=1}^{N_{x}} \sum_{i=0}^{I-1} g_{e, n_{e} n_{u} k} w_{n_{u} n_{x} i} x_{n_{x}}(n-i-k),
$$

80 where the matrix of plant responses, $\mathbf{G}_{e}$, is described by $K$ th order FIR filters with arbitrary accuracy and the impluse response from the $n_{u}$ th secondary source to the $n_{e}$ th virtual error microphone is written as $g_{e, n_{e} n_{u} k}$. Any feedback from the secondary sources to the reference sensors is assumed to be negligable, which can be ensured through the use of feedback cancellation filters, for example. When the $N_{e} N_{u} N_{x}$ filtered-reference signals for the error microphones are written as

$$
r_{e, n_{e} n_{u} n_{x}}(n)=\sum_{k=0}^{K-1} g_{e, n_{e} n_{u} k} x_{n_{x}}(n-k),
$$


85 Eq. (2) can be expressed by substituting Eq. (3) into Eq. (2) as

$$
e_{n_{e}}(n)=d_{e n_{e}}+\sum_{i=0}^{I-1} \boldsymbol{w}_{i}^{\mathrm{T}} \boldsymbol{r}_{e n_{e}}(n-i) \text {, }
$$

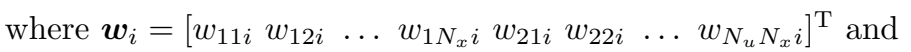

$\boldsymbol{r}_{e n_{e}}=\left[\begin{array}{lllll}r_{e, n_{e} 11} & r_{e, n_{e} 12} & \ldots & r_{e, n_{e} 1 N_{x}} & r_{e, n_{e} 21} \\ r_{e, n_{e} 22} & \ldots & r_{e, n_{e} N_{u} N_{x}}\end{array}\right]^{\mathrm{T}}$. Therefore, the vector of $N_{e}$ error signals, $\boldsymbol{e}(n)=\left[e_{1}(n), e_{2}(n) \ldots e_{N_{e}}(n)\right]^{\mathrm{T}}$ at the virtual error microphones can be expressed as

$$
\boldsymbol{e}(n)=\boldsymbol{d}_{e}(n)+\boldsymbol{R}_{e}(n) \boldsymbol{w}
$$

where

$$
\boldsymbol{R}_{e}(n)=\left(\begin{array}{cccc}
\boldsymbol{r}_{e 1}^{\mathrm{T}}(n) & \boldsymbol{r}_{e 1}^{\mathrm{T}}(n-1) & \cdots & \boldsymbol{r}_{e 1}^{\mathrm{T}}(n-I+1) \\
\boldsymbol{r}_{e 2}^{\mathrm{T}}(n) & \boldsymbol{r}_{e 2}^{\mathrm{T}}(n-1) & \cdots & \boldsymbol{r}_{e 2}^{\mathrm{T}}(n-I+1) \\
\vdots & \vdots & \ddots & \vdots \\
\boldsymbol{r}_{e n_{e}}^{\mathrm{T}}(n) & \boldsymbol{r}_{e n_{e}}^{\mathrm{T}}(n-1) & \cdots & \boldsymbol{r}_{e n_{e}}^{\mathrm{T}}(n-I+1)
\end{array}\right)
$$

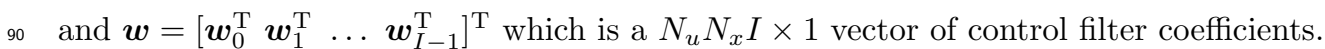

When the direct measurement of the virtual error signals, $\boldsymbol{e}(n)$ is infeasible, they can be estimated from a vector of $N_{m}$ monitoring microphone signals, $\boldsymbol{m}(n)=\left[m_{1}(n), m_{2}(n) \ldots m_{N_{m}}(n)\right]^{\mathrm{T}}$, which is equal to

$$
\boldsymbol{m}(n)=\boldsymbol{d}_{m}(n)+\boldsymbol{R}_{m}(n) \boldsymbol{w},
$$

where $\boldsymbol{d}_{m}(n)=\left[d_{m 1}(n), d_{m 2}(n) \ldots d_{m N_{m}}(n)\right]^{\mathrm{T}}$ is a vector of $N_{m}$ disturbance signals at the monitoring microphones and $\boldsymbol{R}_{m}(n)$ has a similar form to $\boldsymbol{R}_{e}(n)$ in Eq. (6), except that the impluse response from the ${ }_{95} n_{u}$ th secondary source to the $n_{m}$ th monitoring microphone, $g_{m, n_{m} n_{u} k}$, in the plant response, $\mathbf{G}_{m}$, is used in Eq. (3), instead of $g_{e, n_{e} n_{u} k}$.

In practice, the true plant responses, $\mathbf{G}_{e}$ and $\mathbf{G}_{m}$, are not usually available and therefore estimates of the plant responses are used in the controller, as denoted by $\hat{\mathbf{G}}_{e}$ and $\hat{\mathbf{G}}_{m}$. Similarly $\boldsymbol{d}_{m}(n)$ and $\boldsymbol{d}_{e}(n)$ are measured in practice as $\hat{\boldsymbol{d}}_{m}(n)$ and $\hat{\boldsymbol{d}}_{e}(n)$. If $\hat{\boldsymbol{O}}$ is the estimated observation filter used to calculate $\hat{\boldsymbol{d}}_{e}(n)$ 100 from $\hat{\boldsymbol{d}}_{m}(n)$, then the estimated error signals at the virtual error microphones, $\hat{\boldsymbol{e}}(n)$, can be written as

$$
\hat{\boldsymbol{e}}(n)=\hat{\boldsymbol{d}}_{e}(n)+\hat{\boldsymbol{R}}_{e}(n) \boldsymbol{w}(n)=\hat{\boldsymbol{O}} \hat{\boldsymbol{d}}_{m}^{\prime}(n)+\hat{\boldsymbol{R}}_{e}(n) \boldsymbol{w}=\hat{\boldsymbol{O}}\left\{\boldsymbol{m}(n)-\hat{\boldsymbol{R}}_{m}(n) \boldsymbol{w}(n)\right\}^{\prime}+\hat{\boldsymbol{R}}_{e}(n) \boldsymbol{w}
$$

where the superscript - represents an estimate of an actual value and $\hat{O}$ is the matrix of observation filter coef-

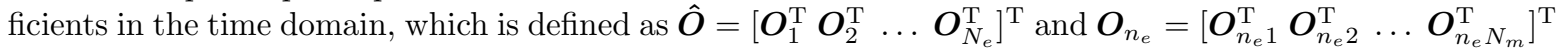
and $\boldsymbol{O}_{n_{e} n_{m}}$ is a vector of coefficients defining the observation filter between the $n_{m}$ th monitoring microphone and the $n_{e}$ th error microphone. This observation filter is modelled by an FIR filter with $J$ coefficients and 105 expressed as

$$
\boldsymbol{O}_{n_{e} n_{m}}=\left[\begin{array}{lllll}
O_{n_{e} n_{m} 0} & O_{n_{e} n_{m} 1} & \ldots & O_{n_{e} n_{m}(J-1)}
\end{array}\right]^{\mathrm{T}},
$$

and $\hat{\boldsymbol{d}}_{m}^{\prime}(n)$ represents the $J N_{m} \times 1$ vector of estimated current and past disturbance signals at the monitoring microphones, which is defined as $\hat{\boldsymbol{d}}_{m}^{\prime}(n)=\left[\hat{\boldsymbol{d}}_{m 1}^{\prime \mathrm{T}}(n) \hat{\boldsymbol{d}}_{m 2}^{\prime \mathrm{T}}(n) \ldots \hat{\boldsymbol{d}}_{m N_{m}}^{\prime \mathrm{T}}(n)\right]^{\mathrm{T}}$ and $\hat{\boldsymbol{d}}_{m n_{m}}^{\prime}(n)=\left[\hat{d}_{m n_{m}}(n) \hat{d}_{m n_{m}}(n-\right.$ 1) $\left.\ldots \hat{d}_{m n_{m}}(n-J-1)\right]^{\mathrm{T}}$ and similarly \{\}$^{\prime}$ represents the $J N_{m} \times 1$ vector, which is built up from the $J$ current and past samples corresponding to the the signals in the braces.

${ }_{110}$ Since the active control system attempts to minimise the estimated error signals, $\hat{\boldsymbol{e}}(n)$, the accuracy of the nearfield estimation using the observation filter, $\hat{\boldsymbol{O}}$, can be an important factor in terms of the actual attenuation at the virtual error microphones. Previous research has shown that when the number of monitoring microphones $\left(N_{m}\right)$ is larger than the number of virtual error microphones $\left(N_{e}\right)$, the problem is mathematically overdetermined and the optimal observation filter, $\boldsymbol{O}_{\mathrm{opt}}$, can be obtained using the derivation 
detailed in Ref. [26] as

$$
\boldsymbol{O}_{\mathrm{opt}}=\left(\left\{E\left[\boldsymbol{d}_{m}^{\prime}(n) \boldsymbol{d}_{m}^{\prime \mathrm{T}}(n)+\beta \mathbf{I}^{\prime}\right]\right\}^{-1} E\left[\boldsymbol{d}_{m}^{\prime}(n) \boldsymbol{d}_{e}^{\mathrm{T}}(n)\right]\right)^{\mathrm{T}},
$$

where $\boldsymbol{d}_{m}^{\prime}(n)$ also represents the $J N_{m} \times 1$ vector of current and past monitoring microphone disturbance signals and $E[]$ is the expectation operator and $\beta$ is a positive real effort-weighting parameter to improve the robustness of the optimal observation filter, and $\mathbf{I}^{\prime}$ is the identity matrix having the same dimensions as $\boldsymbol{d}_{m}^{\prime}(n) \boldsymbol{d}_{m}^{\prime \mathrm{T}}(n)$. The regularisation parameter, $\beta$, is chosen to give a reasonable trade-off between the accuracy with which the disturbance signals are estimated at the virtual microphone positions and the robustness of this estimate to small changes in these positions and to the locations of the primary sources. If $\boldsymbol{d}_{m}^{\prime}(n)$ and $\boldsymbol{d}_{e}(n)$ are measured in preliminary experiments before active control and it is assumed that $\boldsymbol{d}_{m}^{\prime}(n)$ and $\boldsymbol{d}_{e}(n)$ are known perfectly, $\boldsymbol{O}_{\text {opt }}$ can be calculated from Eq. (10) using the measured auto and cross correlation functions of these signals.

The causally constrained optimal controller, $\boldsymbol{w}_{\mathrm{opt}}$, can be derived by minimising the estimated error signals, $\hat{e}$ at the virtual error microphones. In practice, however, it is also necessary to include a term in the cost function that is proportional to the effort required by the control filter to improve the robustness of this filter. Therefore, the cost function, $J_{1}$ can be written as

$$
J_{1}=\operatorname{trace}\left\{E\left[\hat{\boldsymbol{e}}(n) \hat{\boldsymbol{e}}^{\mathrm{T}}(n)+\rho \boldsymbol{w} \boldsymbol{w}^{\mathrm{T}}\right]\right\},
$$

where $\rho$ is a positive real effort-weighting parameter for the controller. To minimise this cost function, it is 130 required that Eq. (8) is expressed in terms of $\boldsymbol{w}$ by substituting Eq. (7) into Eq. (8) to give

$$
\hat{\boldsymbol{e}}(n)=\hat{\boldsymbol{O}} \boldsymbol{d}_{m}^{\prime}(n)+\left[\hat{\boldsymbol{O}} \Delta \boldsymbol{R}_{m}^{\prime}(n)+\hat{\boldsymbol{R}}_{e}(n)\right] \boldsymbol{w},
$$

where $\Delta \boldsymbol{R}_{m}^{\prime}(n) \boldsymbol{w}=\left\{\left[\boldsymbol{R}_{m}(n)-\hat{\boldsymbol{R}}_{m}(n)\right] \boldsymbol{w}\right\}^{\prime}$. Therefore, the causally constrained optimal controller, $\boldsymbol{w}_{\text {opt }}$, with the optimal observation filter, $\boldsymbol{O}_{\mathrm{opt}}$, can be given by $[25,26]$

$$
\boldsymbol{w}_{\mathrm{opt}}=-\left\{E\left[\boldsymbol{R}^{\mathrm{T}}(n) \boldsymbol{R}(n)+\rho \mathbf{I}\right]\right\}^{-1} E\left[\boldsymbol{R}^{\mathrm{T}}(n) \boldsymbol{O}_{\mathrm{opt}} \boldsymbol{d}_{m}^{\prime}(n)\right],
$$

where $\boldsymbol{R}(n)=\left[\boldsymbol{O}_{\text {opt }} \Delta \boldsymbol{R}_{m}^{\prime}(n)+\hat{\boldsymbol{R}}_{e}(n)\right]$ and $\mathbf{I}$ is the identity matrix having the same dimensions as $\boldsymbol{R}^{\mathrm{T}}(n) \boldsymbol{R}(n)$.

\subsection{Optimal feedforward active control using the delayed remote microphone technique}

When the monitoring microphones can detect the disturbance signals in advance of the virtual error microphones, the causality between the virtual error microphones and the monitoring microphones can 135 be maintained and the standard remote microphone technique in the previous section can be applied to estimate the virtual error signals accurately. However, when the disturbance signals arrive at the virtual error microphones earlier than at the monitoring microphones, due to the relative geometry of the virtual error microphones, the monitoring microphones and the primary sources, the causality between the virtual error and monitoring microphones would be significantly degraded. This is because the estimation of 'future' 140 random signals at the virtual error microphones from the monitoring microphones may not be achievable.

To overcome this limitation, an improved remote microphone technique called the 'delayed' remote microphone technique, suggested in [23], is applied to the feedforward active control algorithm, as shown in Fig. 2. In the delayed remote microphone technique, the vector of $N_{m}$ monitoring microphone signals, $\boldsymbol{m}(n)$, is used to estimate the disturbance signals at the virtual error microphones with a delay of $\Delta$ samples, ${ }_{145} \hat{\boldsymbol{d}}_{e}(n-\Delta)$ with the delayed optimal observation filter, $\boldsymbol{O}_{\mathrm{opt}, \mathrm{D}}$ which is given by

$$
\boldsymbol{O}_{\mathrm{opt}, \mathrm{D}}=\left(\left\{E\left[\boldsymbol{d}_{m}^{\prime}(n) \boldsymbol{d}_{m}^{\prime \mathrm{T}}(n)+\beta \mathbf{I}^{\prime}\right]\right\}^{-1} E\left[\boldsymbol{d}_{m}^{\prime}(n) \boldsymbol{d}_{e}^{\mathrm{T}}(n-\Delta)\right]\right)^{\mathrm{T}} .
$$

By minimising the cost function $J_{2}=\operatorname{trace}\left\{E\left[\hat{\boldsymbol{e}}(n-\Delta) \hat{\boldsymbol{e}}^{\mathrm{T}}(n-\Delta)+\rho \boldsymbol{w} \boldsymbol{w}^{\mathrm{T}}\right]\right\}$, the causally con- 


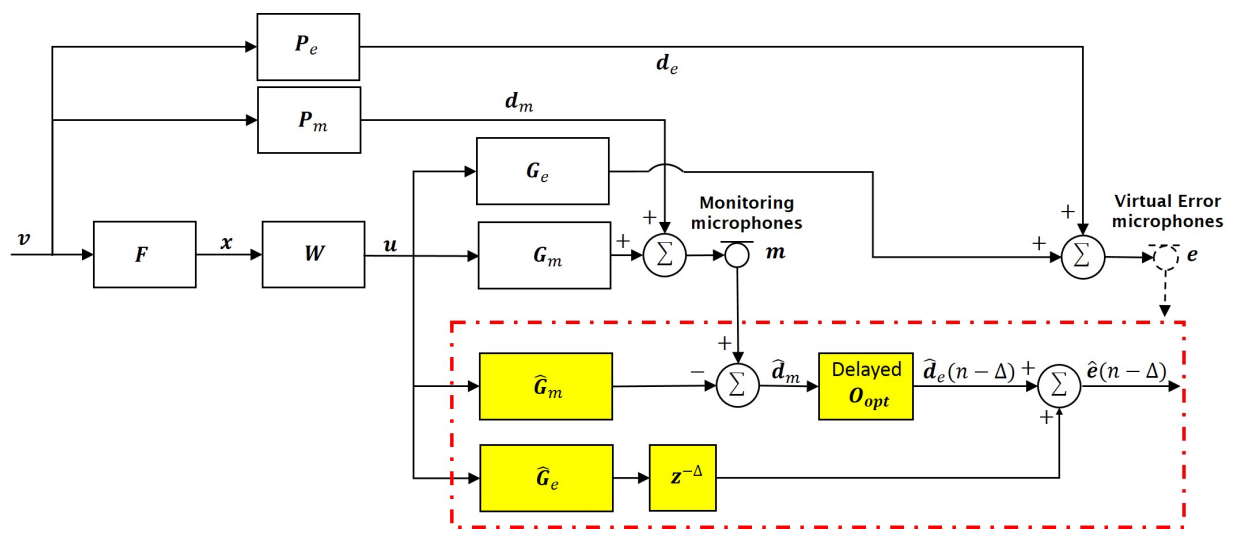

Figure 2: Block diagram of the feedforward active control algorithm, combined with the delayed remote microphone technique in a dashed dot rectangle.

strained optimal controller with the delayed remote microphone technique, $\boldsymbol{w}_{\mathrm{opt}, \mathrm{D}}$ is given by

$$
\boldsymbol{w}_{\mathrm{opt}, \mathrm{D}}=-\left\{E\left[\boldsymbol{R}_{\mathrm{D}}^{\mathrm{T}}(n) \boldsymbol{R}_{\mathrm{D}}(n)+\rho \mathbf{I}\right]\right\}^{-1} E\left[\boldsymbol{R}_{\mathrm{D}}^{\mathrm{T}}(n) \boldsymbol{O}_{\mathrm{opt}, \mathrm{D}} \boldsymbol{d}_{m}^{\prime}(n)\right],
$$

where $\boldsymbol{R}_{\mathrm{D}}(n)=\left[\boldsymbol{O}_{\text {opt,D }} \Delta \boldsymbol{R}_{m}^{\prime}(n)+\hat{\boldsymbol{R}}_{e}(n-\Delta)\right]$. When the disturbance signals from the primary sources are stationary, $\boldsymbol{w}_{\text {opt,D }}$ is almost identical to $\boldsymbol{w}_{\text {opt }}$ and active control at the virtual error microphones can still be achieved. The selection of $\Delta$ in practice is discussed in the following section.

\section{Remote microphone technique inside a vehicle}

\subsection{Experimental arrangement}

To estimate the effect of local active sound control with the remote microphone technique in a vehicle, an active headrest system with a dummy head was installed at the rear right seat of a large SUV, as shown in 155 Fig. 3. In addition, 16 monitoring microphones were installed around the dummy head and Fig. 3 also shows how these 16 monitoring microphones, located at different positions in the vehicle cabin, are numbered for reference. The interior noise at the monitoring microphones and the virtual error microphones at the ears of the dummy head were measured when the car was driven over a rough road at $80 \mathrm{kph}$, and then used to calculate the observation filters and to analyse the accuracy of the standard and delayed remote microphone 160 techniques. To compare the estimated disturbance signals at the virtual error microphones with the actual disturbance signals, two microphones in the ears of the dummy head were used in these measurements as the physical error sensors. The sampling frequency was $3 \mathrm{kHz}$ and a series of experiments was conducted when the dummy head was located at 7 different positions on a $5 \times 4$ grid of points spaced $5 \mathrm{~cm}$ apart, as shown in Fig. 4, to investigate the potential accuracy of the remote microphone technique and the achievable 165 attenuation of the active headrest system when a listener's head is moved.

\subsection{Nearfield estimation of interior noise at desired positions using arrays of monitoring microphones}

To conduct the nearfield estimation of interior noise at the ears of the dummy head, the optimal observation filters for the standard and delayed remote microphone techniques were calculated using Eq. (10) and Eq. (14) using the auto and cross correlation functions calculated from the measured disturbance signals. ${ }_{170}$ The normalised mean squared estimation error level at a single virtual error microphone, $L_{\epsilon}$, can be defined as

$$
L_{\epsilon}=10 \log _{10}\left|\frac{S_{\epsilon \epsilon}}{S_{d_{e} d_{e}}}\right|
$$


(a)
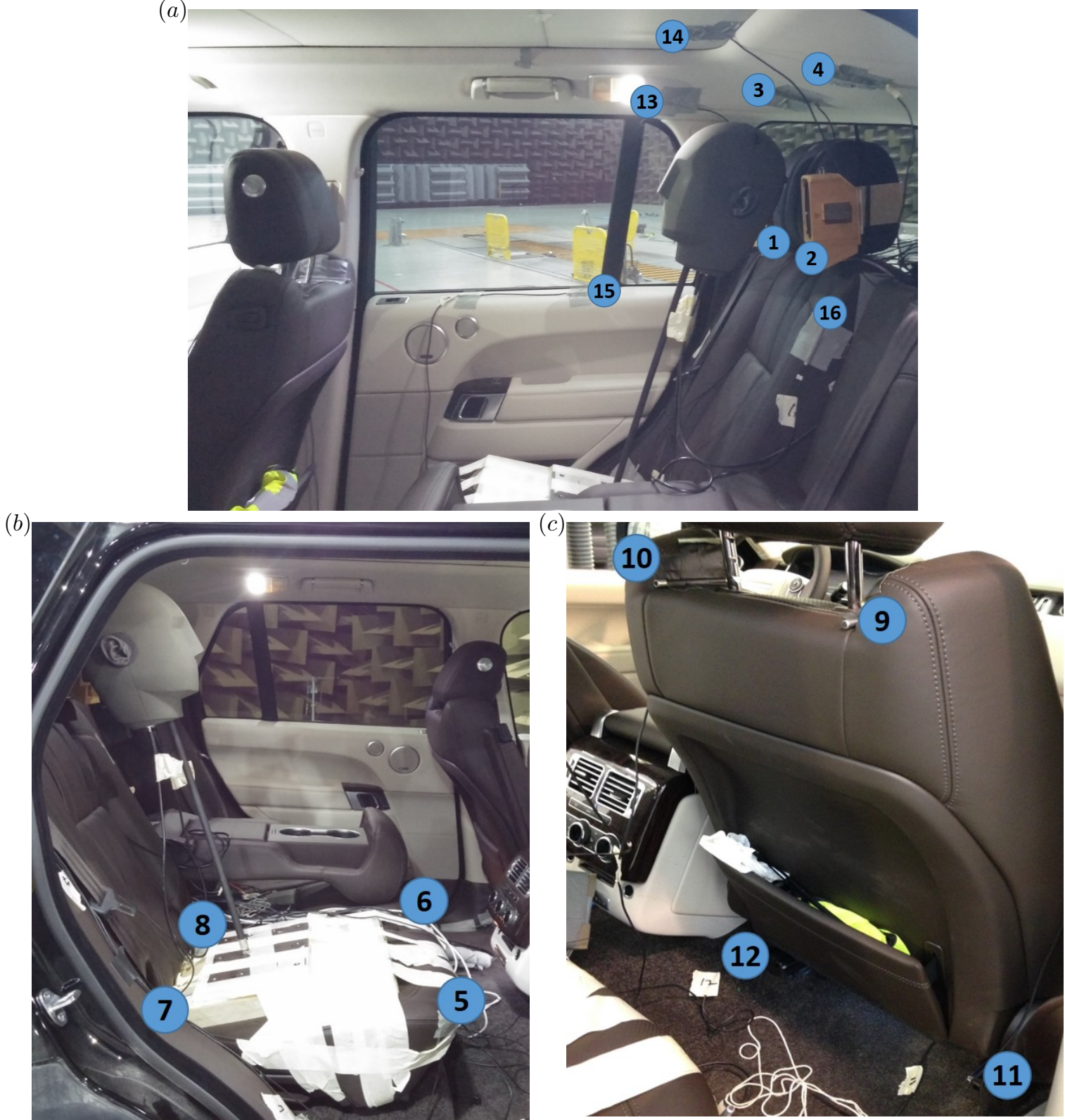

Figure 3: The overall installation for the remote microphone technique and local active sound control experiments when interior road noise is measured at the rear right seat of a large SUV using 16 potential positions for monitoring microphones around the dummy head and two error microphones in the ears of a dummy head. Eight potential monitoring microphones are positioned on the headrest and ceiling (a), four monitoring microphones on the rear right seat (b) and four monitoring microphones on the front right seat (c). 


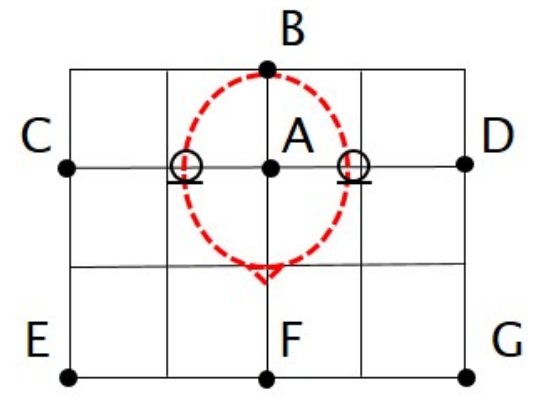

Figure 4: Location of the different head positions on the measurement grid.

where $\epsilon(n)$ is defined as $d_{e}(n)-\hat{d}_{e}(n)$ at a single virtual microphone location and $S_{\epsilon \epsilon}$ and $S_{d_{e} d_{e}}$ are the power spectral densities (PSDs) for $\epsilon(n)$ and $d_{e}(n)$, respectively.

Fig. 5 shows examples of the nearfield estimation error when different sets of monitoring microphones 175 are selected from the overall array in Fig. 3. The causally constrained optimal observation filters were implemented using FIR filters with $J=256$ filter coefficients and the dummy head was located in the nominal position 'A', as shown in Fig. 4. For the delayed remote microphone technique, a delay of $\Delta=15$ samples was included, which was a sufficient delay to allow important non-causal components of responses to be included. This delay corresponds to a sound propagation distance of about $1.7 \mathrm{~m}$. To provide a benchmark

180 for the best possible estimation accuracy with this microphone arrangement, the nearfield estimation was also conducted for a non-causal optimal observation filter in the frequency domain, as suggested by [26]. No regularisation factors were required for the observation filters for the results in Fig. 5, because they were effectively already introduced as a result of the measurement uncertainty and the condition number of inverted terms in the observation filters were already sufficiently small. It can be seen that although 185 the nearfield estimation error is variable due to the complexity of the acoustic field, the error generally increases as the frequency increases. As expected, the best nearfield estimation is achieved with the noncausal frequency domain filters, which do not enforce any constraints on the causality or the number of filter coefficients, but the causal filters do achieve a similar accuray to the non-causal filter.

When all the 16 monitoring microphones were selected for the estimation, the result in Fig. 5 (b) shows 190 that using the remote microphone techniques, the disturbance signals at the right ear can be estimated with less than $-10 \mathrm{~dB}$ estimation error up to around $800 \mathrm{~Hz}$, except at certain frequencies but the nearfield estimation error at the left ear in Fig. 5 (a) is generally higher than at the right ear. This is thought to be because sound waves directly propagated from the rear right wheel of the vehicle are dominantly measured at the right ear and the monitoring microphones. Lower correlation between the rear right wheel and the left

195 ear is produced due to the further distance between the rear right wheel and the left ear and the scattering effect of the dummy head. The eight monitoring microphones used for the results in Fig. 5(c) and (d) were selected from the 16 monitoring microphones through an exhaustive search to give the best performance and it was found that the best positions were with four microphones (\#1 \#4) on and around the headrest and four microphones (\#13 \#16) above and both sides of the dummy head. In a similar way, only four 200 monitoring microphones were selected using an exhaustive search with the results shown in Fig. 5(e) and (f) as two microphones (\#1 and \#2) on the headrest and two microphones (\#13 and \#14) above and in the right of the dummy head. The results in Fig. $5(\mathrm{c}) \sim(\mathrm{f})$ show that although the nearfield estimation error is degraded by the use of a smaller number of monitoring microphones, $-10 \mathrm{~dB}$ estimation error is achieved up to about $400 \mathrm{~Hz}$ even with only 4 monitoring microphones. These results indicate that the remote 205 microphone technique for the active headrest system can achieve accurate nearfield estimation for active sound control at low frequencies with a small number of monitoring microphones, but at higher frequencies, larger number of monitoring microphones are necessary to allow good attenuation.

It can also be seen in Fig. 5 that somewhat better nearfield estimation with the delayed remote mi- 
(a)

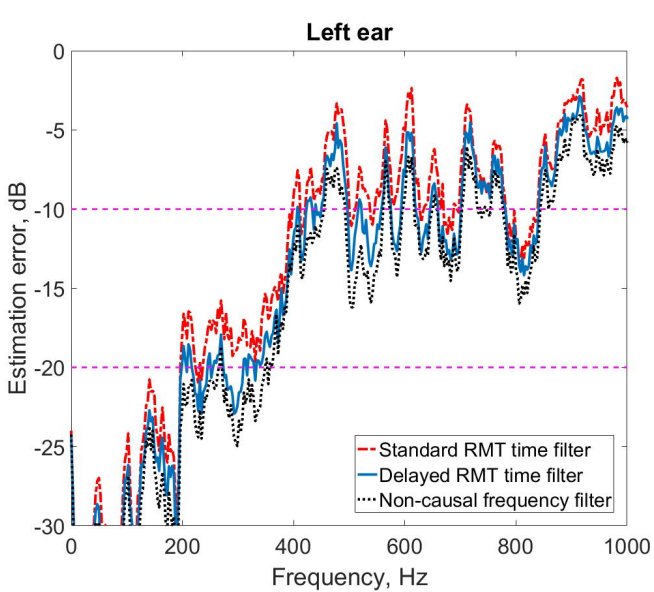

(c)

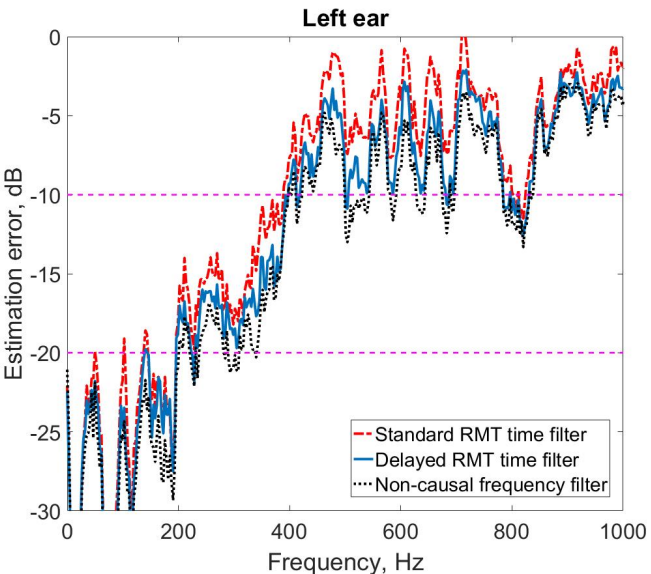

(e)

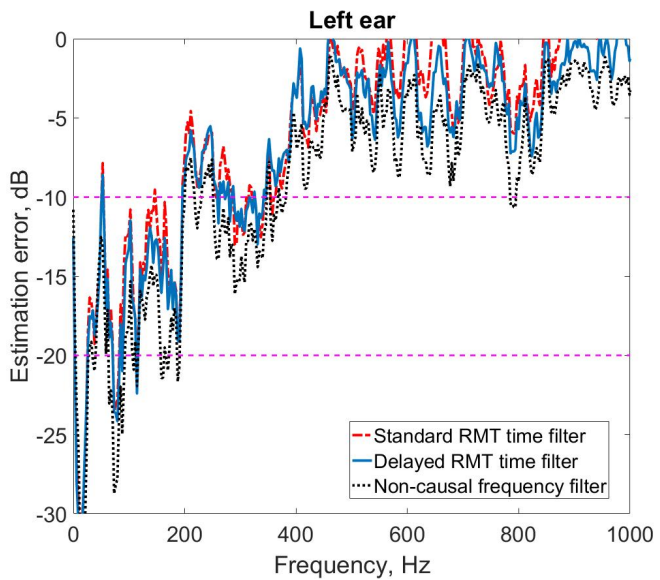

(b)

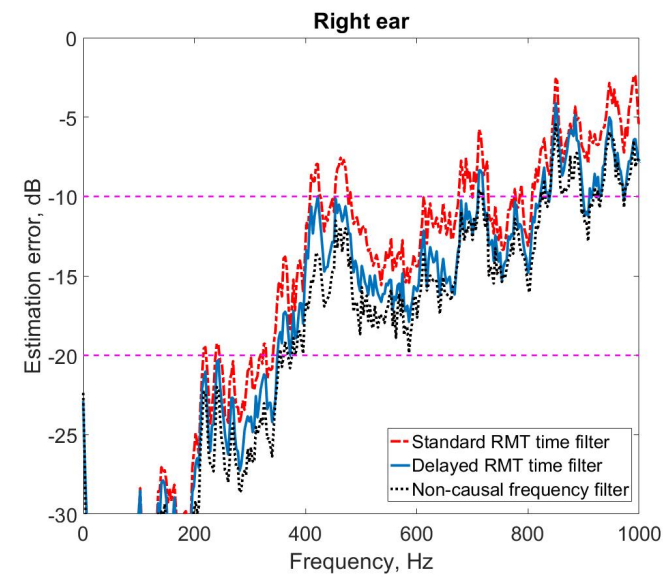

(d)

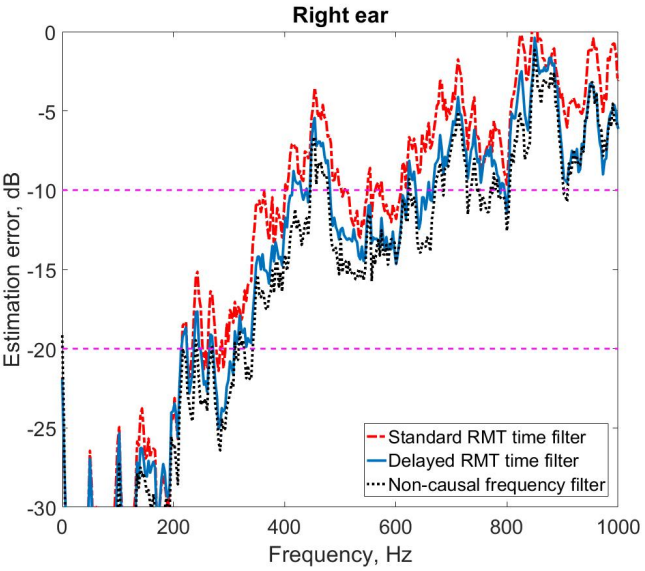

(f)

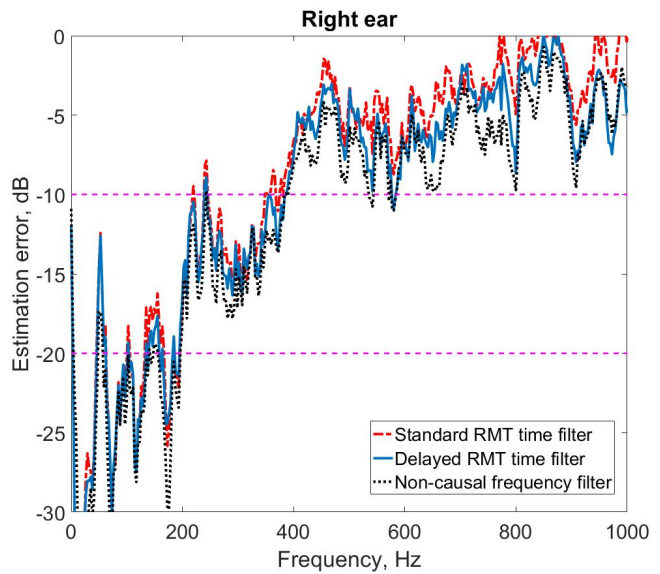

Figure 5: The nearfield estimation error of the remote microphone technique (RMT), calculated from data measured on a rough road at $80 \mathrm{kph}$ when different arrays of monitoring microphones are selected from the array in Fig. 3 to estimate the interior road noise at the ears of the dummy head: (a) and (b) all 16 monitoring microphones, (c) and (d) 8 monitoring microphones \#1 \#4, \#13 \#16 and (e) and (f) 4 monitoring microphones \#1, \#2, \#13, \#14. 
crophone technique is achieved, compared to the standard remote microphone. This is probably because 210 in the vehicle cabin, important non-causal components are present, due to both the complex distribution of primary source excitations and the reverberant nature of the cabin. The effect of the delayed remote microphone technique can also be investigated by comparing the impulse responses of the observation filters with those calculated using the standard method in the frequency domain. These responses are shown in Fig. 6 for one of the 32 impulse responses, $\boldsymbol{O}_{11}$, calculated when all 16 potential monitoring microphones ${ }_{215}$ are used to estimate the disturbance at the right ear of the dummy head. In Fig. 6(a), it can be seen that the impulse response corresponding to the causally constrained time domain filter calculated using the standard remote microphone technique is similar to the causal part of the inverse Fourier transformed optimal observation filter calculated in the frequency domain but the non-causal part of this response is not present in the causally constrained filter. In Fig. 6(b), however, it can be seen that by using the delayed remote

220 microphone technique, the important non-causal part of the response is included in the impulse response, which results in the improved nearfield estimation.

When the head position of a listener is changed, pre-calculated optimal observation filters can be updated by position information from an optical head tracking device, as suggested by [21, 22]. Therefore, the availability of the remote microphone technique for different head positions is investigated as the dummy

225 head is located at either position ' $\mathrm{E}$ ' or ' $\mathrm{G}$ ' in Fig. 4 and the nearfield estimation error with the overall 16 monitoring microphones is calculated as shown in Fig. 7. By comparing Fig. 7 with Fig. 5, it can be seen that although the dummy head is moved around $14 \mathrm{~cm}$ away from the nominal position, the accuracy of the nearfield estimation can be maintained with fixed monitoring microphones installed around the dummy head.

\section{4. Active control of road noise inside a vehicle}

\subsection{Attenuation performance of the mutichannel active headrest system}

In the previous section, the accuracy of the remote microphone technique (RMT) in the vehicle has been investigated. It is also interesting to predict the potential attenuation performance of the active system in a real vehicle cabin. Therefore, this section presents off-line analysis results of the attenuation

235 performance obtained with the same installation used in previous section. The plant responses between two loudspeakers mounted in the active headrest system and the monitoring and error microphones, $\mathbf{G}_{m}$ and $\mathbf{G}_{e}$, have been measured and were modelled by $K=64$ th order FIR filters. Reference signals from eight sensors installed around the four wheels were simultaneously measured along with interior disturbance signals when the car was driven at $80 \mathrm{kph}$ on a rough road. For simplicity of calculation, it is assumed 240 that the true plant responses, $\mathbf{G}_{m}$ and $\mathbf{G}_{e}$ are identical to the estimated plant responses, $\hat{\mathbf{G}}_{e}$ and $\hat{\mathbf{G}}_{m}$, resulting in $\boldsymbol{R}(n)=\boldsymbol{R}_{e}(n)$ in Eq. (13) and $\boldsymbol{R}_{\mathrm{D}}(n)=\boldsymbol{R}_{e}(n-\Delta)$ in Eq. (15). With the pre-calculated optimal observation filters presented in the previous section, the optimal controllers, which were modelled using the $I=2,048$ th order FIR filters, were calculated using Eq. (13) and Eq. (15). As a benchmark for the best possible performance of the causal controller with this arrangement, a controller was also calculated 245 using disturbance signals from the dummy head microphones directly and using $\boldsymbol{d}_{e}(n)$ in Eq. (13), instead of $\boldsymbol{O}_{\mathrm{opt}} \boldsymbol{d}_{m}^{\prime}(n)$.

To find a suitable regularisation factor, $\rho$ in Eq. (13) and Eq. (15), the averaged attenuation levels up to $1 \mathrm{kHz}$ for different regularisation factors have been calculated from the sums of the power spectral densities (PSDs) of the signals at each physical error microphone in the dummy head before and after control, as

${ }_{250}$ shown in Fig. 8. It can be seen that with smaller regularisation factors, below $10^{-3}$, the attenuation level is negative, i.e. an enhancement is predicted, since the inverted term in Eq. (13) and Eq. (15) is very sensitive to numerical uncertainties and with larger regularisation factors, above $10^{-3}$, the attenuation level is reduced due to the biased solution.

With a regularisation factor of $\rho=10^{-3}$, the optimal performance of the three causal controllers (the 255 controller with direct measurement from the physical error microphones, the controller with the standard RMT and the controller with the delayed RMT) has been calculated, using the A-weighted PSDs of the signals at the headrest error microphones, using the plant responses, the interior road noise signals measured 
(a)

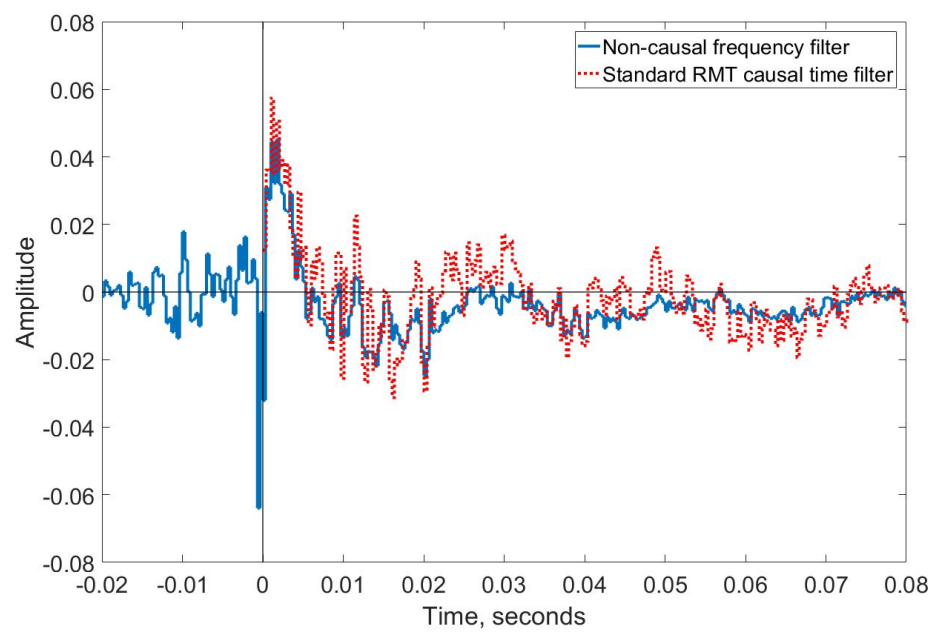

(b)

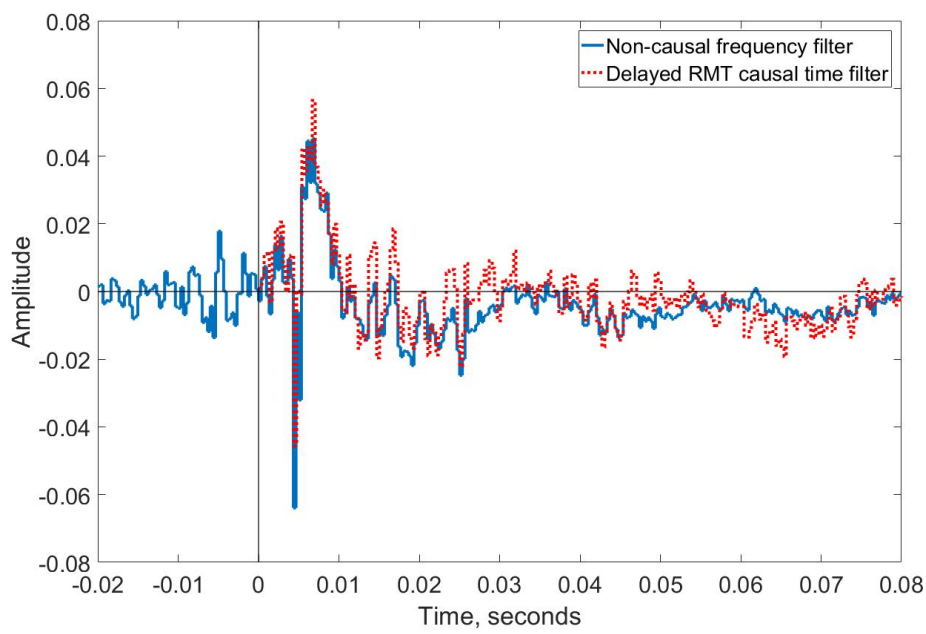

Figure 6: Comparison of the impulse response of $\boldsymbol{O}_{11}$ among the FIR filters of the causal constrained time filter with the inverse Fourier transformed $\mathbf{O}_{11}$, which is among responses of the non-causal optimal observation filter in the frequency domain when the 16 monitoring microphones were used to estimate disturbance signals at the right ear of the dummy head when driven at $80 \mathrm{kph}$ on a rough road: (a) the standard remote microphone technique, (b) the delayed remote microphone technique. 
$(a)$

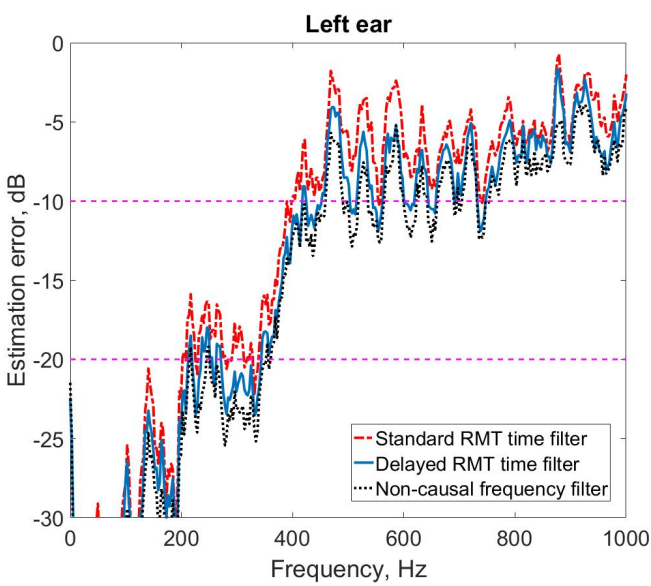

$(c)$

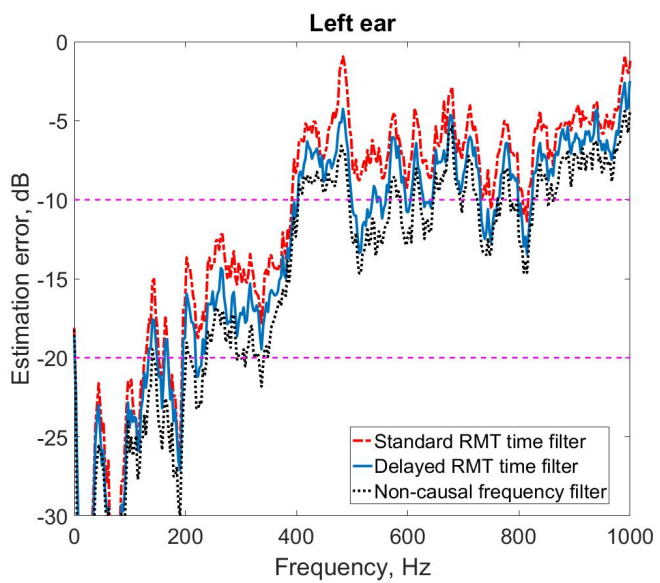

$(b)$

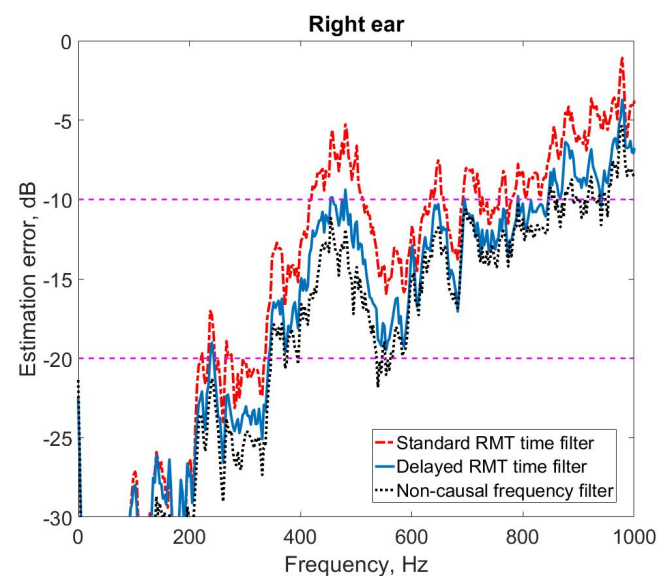

$(d)$

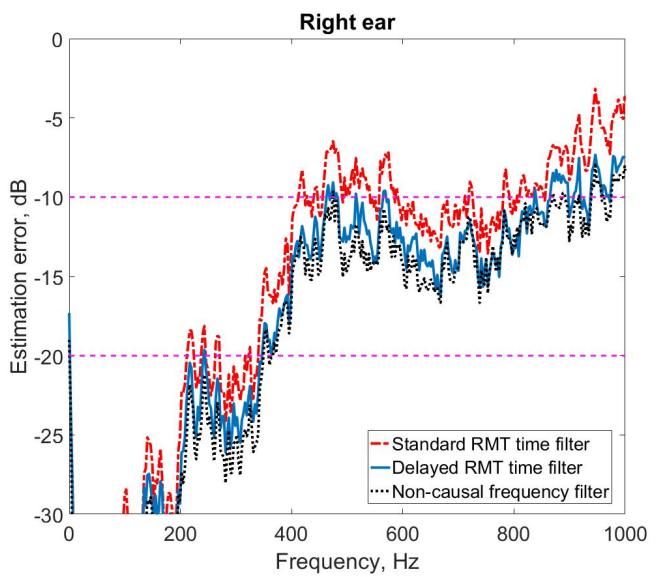

Figure 7: The nearfield estimation error for different head positions in Fig. 4, calculated from data measured on a rough road at $80 \mathrm{kph}$ when 16 monitoring microphones are used: (a) and (b) head position 'E' and (c) and (d) head position 'G'. 


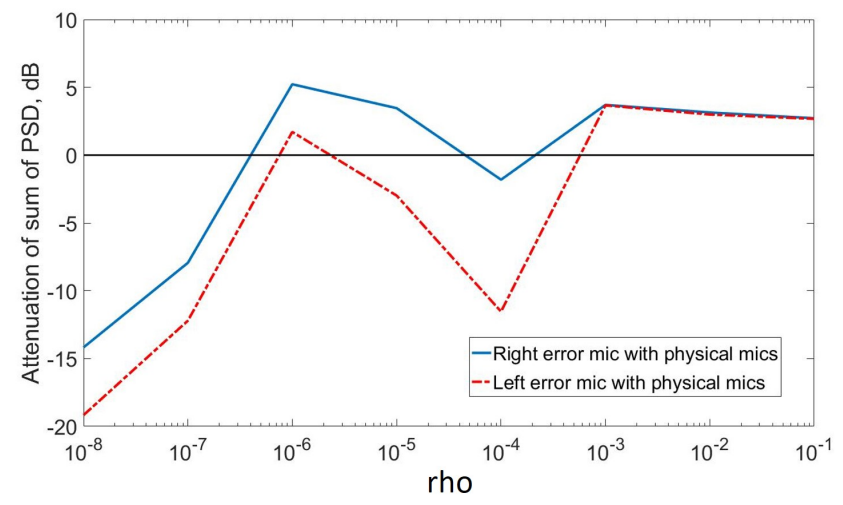

Figure 8: Averaged attenuation levels for different regularisation factors, $\rho$ in Eq. (13) and Eq. (15), obtained from the sums of the PSDs of signals at the physical error microphones when disturbance signals at the dummy head on the position 'A' of the grid and reference signals are measured on a rough road surface at $80 \mathrm{kph}$ and the active headrest system with two secondary loudspeaker is used to reduce noise.

at the 16 monitoring microphones and two error microphones, and reference sensor signals when the car is driven at $80 \mathrm{kph}$ on a rough road surface. In addition, the optimal performance of a non-causal controller 260 using the physical error microphones has been calculated from the frequency responses with the same installation, using the solution given in $[25,26]$. Previous results have shown that the optimal attenuation of the non-causal controller for feedforward active control has an upper lmit determined by the multiple coherence function, $\gamma^{2}$, between the reference sensors and the error sensors as [25]

$$
\text { Att }=10 \log _{10}\left(1-\gamma^{2}\right)
$$

The uncontrolled and controlled disturbances are presented in Fig. 9. It can be seen in Fig. 9(a) and 265 (b) that the causal controller with the physical error microphones can effectively attenuate several peaks of broadband interior noise up to $1 \mathrm{kHz}$ with a maximum reduction at $747 \mathrm{~Hz}$ of $8.6 \mathrm{~dB}$ at the right ear and an average reduction of around $3.7 \mathrm{~dB}$ which is similar to that of the non-causal controller, although attenuation levels with these controllers at discrete frequency ranges are different from other. Fig. 9(c) and (d) show that the causal controllers with the different RMTs can also reduce the broadband interior noise 270 with a similar level of attenuation to that achieved by the controllers with the physical error microphones in Fig. 9(a) and (b) because the virtual error signals are quite accurately estimated using the observation filters in Fig. 5(a) and (b). Although the delayed RMT provides more accurate nearfield estimation, compared to the standard RMT in Fig. 5(a) and (b), the attenuation performance with these two RMT methods are similar to each other because the performance in this case is limited by the coherence between the reference 275 and disturbance signals, rather than the errors inherent in the RMT estimation. In other experiments, it was found that when the coherence between the reference and disturbance signals is greater, there is a clear difference in the active control performance with the standard and delayed RMT.

\subsection{Effects of time delays and head movements on performance}

For the practical application of the active headrest system, the effect of processing delays should be considered because although delays from the digital-to-analogue and analogue-to-digital converters (DAC and $\mathrm{ADC}$ ) and the anti-aliasing and reconstruction filters were already included in the measured signals and transfer responses, in a real-time system additional delays can be generated by the processing time needed to calculate the control signals. Previous research has shown that the attenuation performance can be degraded by these delays $[6,8,27]$. The effect of the delays on the overall reduction of the active headrest system 285 with the physical error microphones is shown in Fig. 10. It can be seen that as the delay is increased, the 
(a)

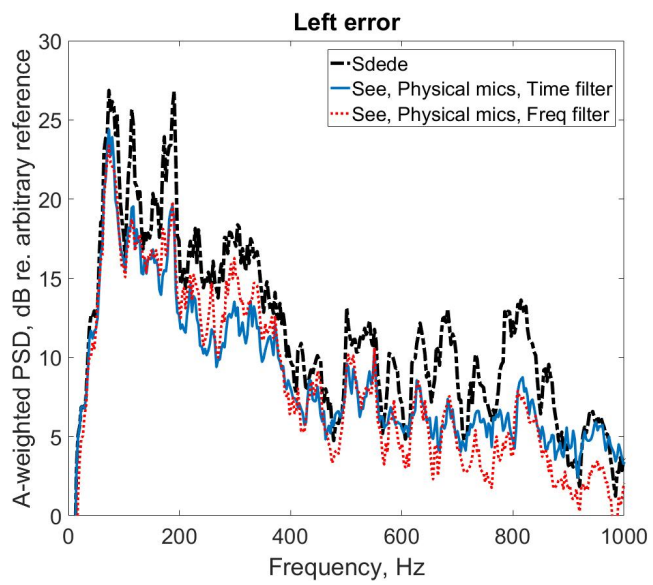

(c)

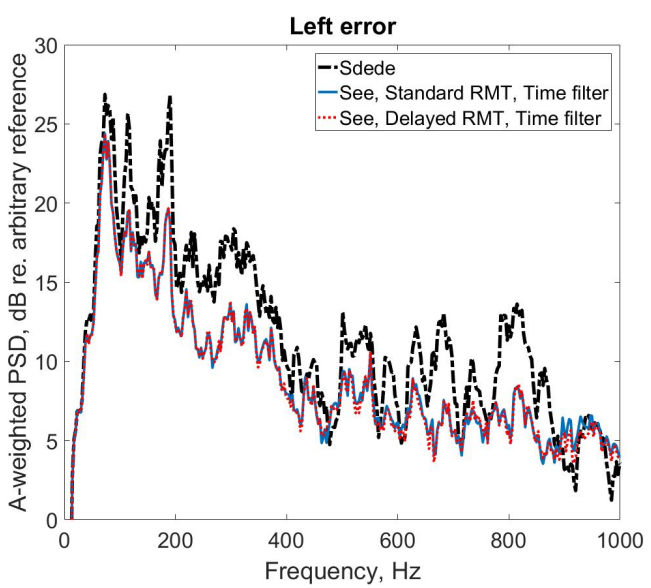

(b)

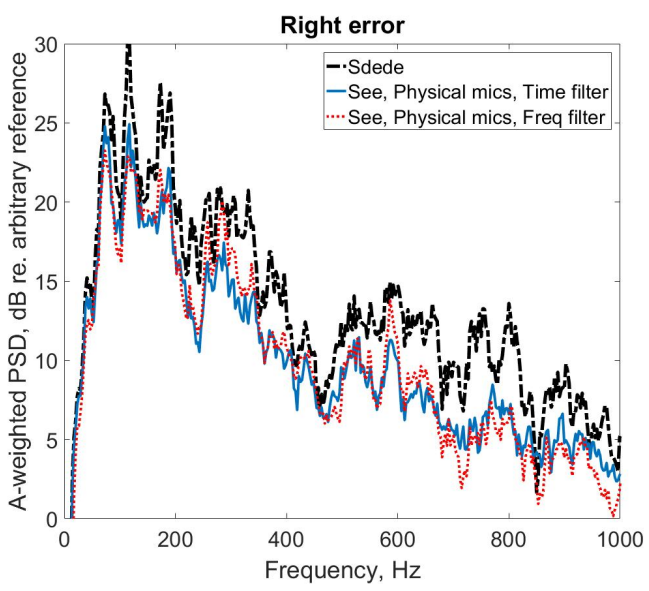

(d)

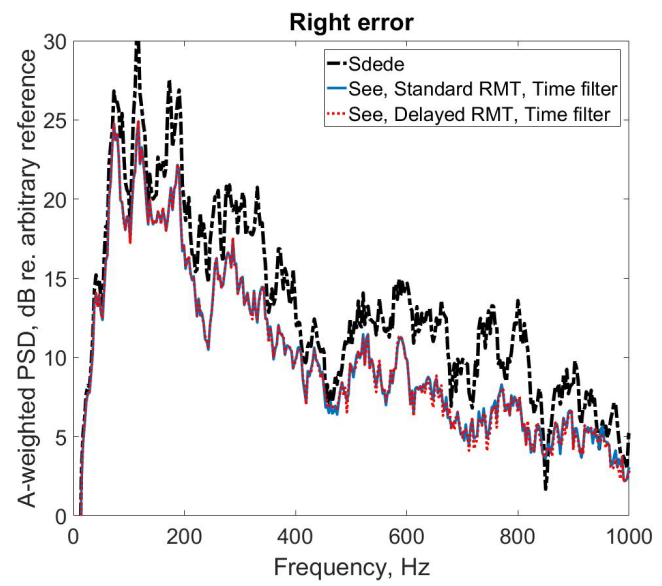

Figure 9: A-weighted PSDs of the signals at the left and right error microphones of the dummy head before and after control, $S_{d_{e} d_{e}}$. Simulations of control calculated using the plant responses, the interior road noise signals measured at the 16 monitoring microphones and two error microphones, and reference sensor signals when the car is driven at $80 \mathrm{kph}$ on a rough road surface: (a) and (b) Predicted performances assuming physical error sensors be used in the ears of the dummy head. The uncontrolled disturbances (dot-dashed lines), the controlled disturbances by the causal time filter with the physical error microphones (solid lines) and the controlled disturbances by the non-causal frequency filter with the physical error microphones (dotted lines), (c) and (d) Predicted performances assuming the causal time filters for the standard and delayed RMT be used. The uncontrolled disturbances (dot-dashed lines), the controlled disturbances by the causal time filter with the standard RMT (solid lines) and the controlled disturbances by the causal time filter with the delayed RMT (dotted lines). 


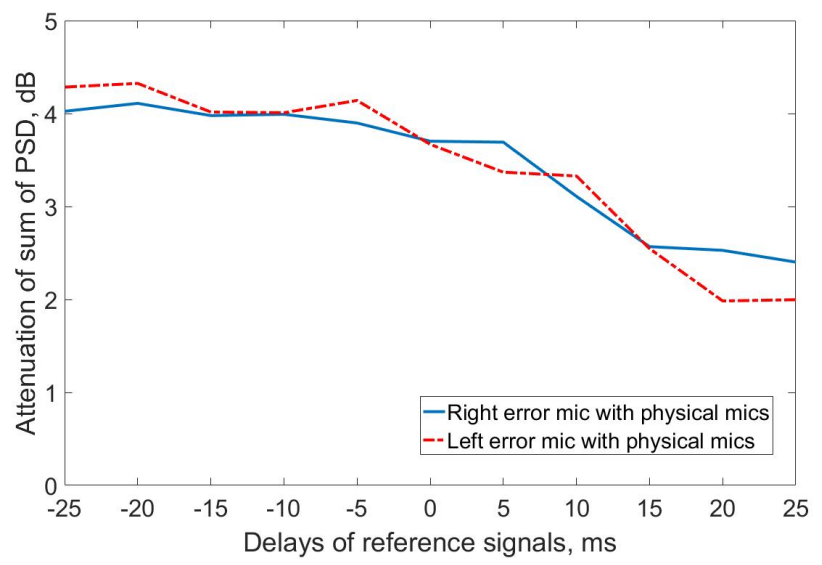

Figure 10: Averaged attenuation levels when various delays are applied for the feedforward active headrest system.

attenuation performance is progressively decreased and delays of more than $10 \mathrm{~ms}$ only degrade attenuation performance by about $1 \mathrm{~dB}$ in this case. Conversely, if the inherent delays from DAC and ADC and the anti-aliasing and reconstruction filters are reduced, more time-advanced reference signals would be available, which can be modelled as a negative processing delay in Fig. 10, and the attenuation performance can thus 290 be slightly improved.

In the previous section, when the position of the dummy head is changed, the nearfield estimation using the RMTs was investigated and the results have shown that when the optimal observation filter is updated to that for the corresponding head position, the performance of the nearfield estimation can be maintained with the fixed monitoring microphones. Similarly, when pre-modelled plant responses are updated for the 295 perturbed head positions, the attenuation performance of the active headrest system is shown in Fig. 11. It can be seen that when the dummy head is moved to position ' $E$ ' or ' $G$ ' in Fig. 4, the overall attenuation up to $1 \mathrm{kHz}$ is similar to that achieved at position ' $\mathrm{A}$ '. The attenuation with the Delayed RMT is similar to that achieved with the physical error microphones, due to the accurate nearfield estimation, as seen in the previous section. From these results, it is clear that as the head-tracking system updates the pre-modelled 300 observation filters and plant responses, the controlled zones of the active headrest system can move with the ear positions as if the user was wearing active sound control headphones.

\section{Conclusion}

Local active sound control with an active headrest system may be an effective method to reduce broadband random road noise in a vehicle cabin at frequencies higher than can be controlled with a global active 305 control system, using more distributed loudspeakers and microphones. Since the direct measurement of the error signals at the ears of a listener is not practical, we have investigated the potential accuracy of the remote microphone technique in this application, to estimate the error signals at the ear positions. The potential performance of the active headrest system in controlling random road noise has also been investigated, using the remote microphone technique. The optimal observation filter for the remote microphone

310 technique, and the corresponding optimal controller with a causality constraint, have been formulated in the time domain, to sense and control broadband random disturbances. To improve the causality of the remote microphone technique, a formulation for the delayed remote microphone technique has also been used to estimate the delayed signals at the virtual error microphones, and the optimal controller with this technique has also been formulated.

315 The performance of the remote microphone technique in the vehicle cabin has been investigated by processing the disturbance signals measured at 16 monitoring microphones around the cabin and two error 
$(a)$

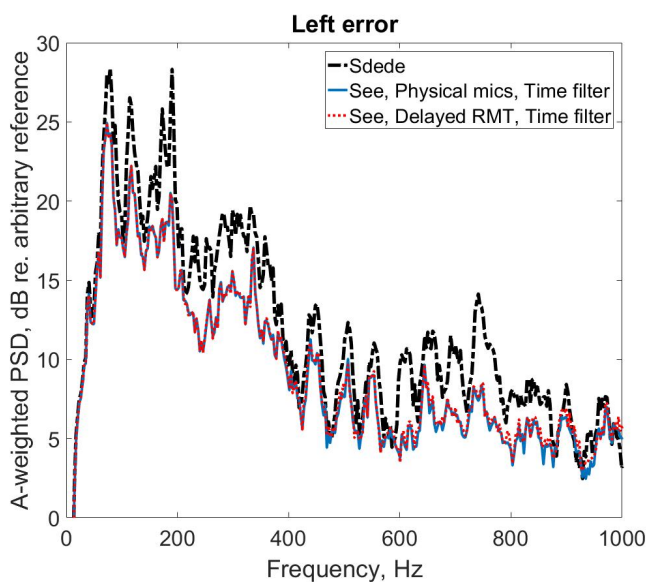

$(c)$

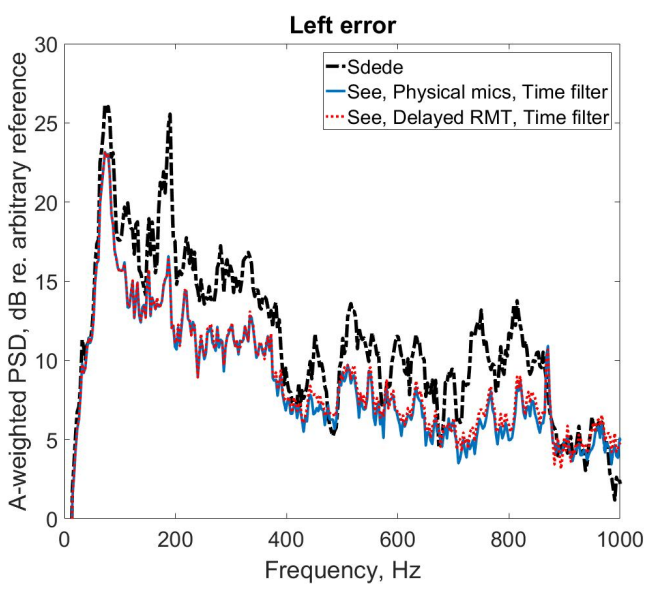

$(b)$

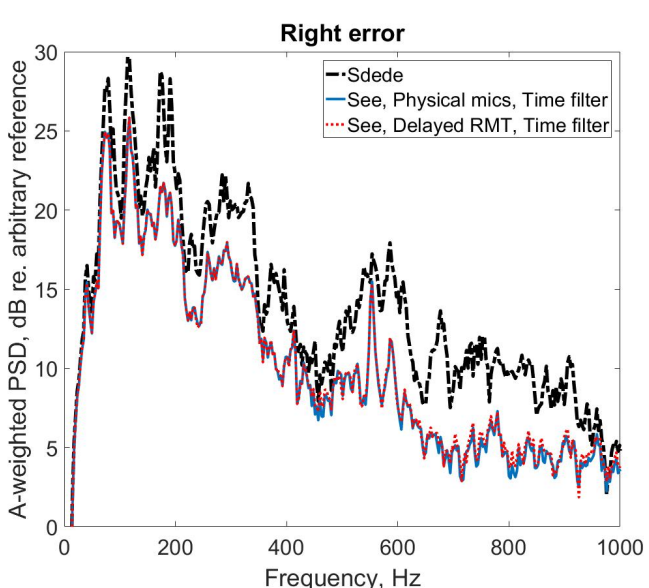

$(d)$

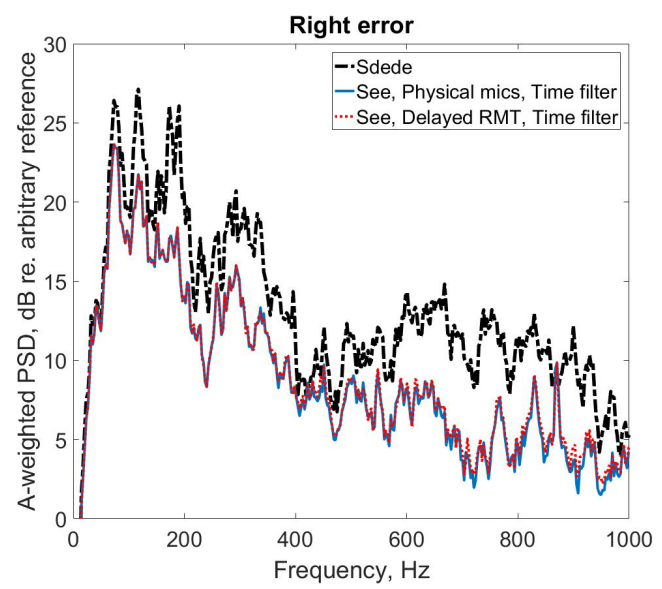

Figure 11: The A-weighted PSDs of signals at the left and right error microphones for different head positions in Fig. 4: (a) and (b) head position 'E' and (c) and (d) head position 'G'. 
microphones at the ears of a dummy head, when the car was driven over a rough road at $80 \mathrm{kph}$. The calculated nearfield estimation error between the actual and estimated disturbances at the error microphones has shown that with all 16 monitoring microphones, an estimation error of less than $-10 \mathrm{~dB}$ is achieved up

320 to $800 \mathrm{~Hz}$ at the right ear whereas with only four monitoring microphones, this estimation error is only achieved up to $400 \mathrm{~Hz}$. When the dummy head is moved to different positions, the results demonstrate that if a pre-modelled observation filter is updated using position information, from a head tracking device for example, the signals at the perturbed virtual error sensors can still be estimated using fixed monitoring sensors.

The performance of a multichannel feedforward active headrest system combined with the remote microphone technique has been predicted offline using disturbance signals at the monitoring and error microphones, plant responses and reference signals measured in the car. The causal controller achieves a broadband average reduction of around $3.7 \mathrm{~dB}$ up to $1 \mathrm{kHz}$ and a maximum reduction of $8.6 \mathrm{~dB}$ is achieved at $747 \mathrm{~Hz}$ at the right ear. The attenuation performance with the remote microphone technique is similar

330 to that obtained if the pressure at the ears of the dummy head used in these experiments were directly controlled, illustrating the potential for using remote monitoring microphones. When the dummy head is moved to different positions, the simulation results show that good attenuation at the ears can still be achieved by updating pre-modelled plant responses and observation filters if the position of the head is always known.

\section{Acknowledgments}

335 This research is jointly funded by an EPSRC industrial CASE studentship (Award no. 14220108) with Jaguar Land Rover (JLR). The authors are especially thankful to Dr Delphine Nourzad and Mr Xavier Vinamata of JLR for their support as industrial supervisors.

\section{Additional information}

Declarations of interest: none.

\section{References}

[1] N. Lalor, H. H. Priebsch, The prediction of low-and mid-frequency internal road vehicle noise: a literature survey, in: Proceedings of the Institution of Mechanical Engineers, Part D: Journal of Automobile Engineering, Vol. 221, Sage Publications, 2007, pp. 245-269.

[2] D. J. Thompson, J. Dixon, Vehicle noise, in: F. Fahy (Ed.), Advanced applications in acoustics, noise and vibration, CRC Press, Florida, USA, 2004, Ch. 6, pp. 236-291.

[3] X. Wang, Vehicle Noise and Vibration Refinement, Elsevier, Amsterdam, 2010.

[4] S. J. Elliott, Active noise and vibration control in vehicles, in: X. Wang (Ed.), Vehicle Noise and Vibration Refinement, Elsevier, Amsterdam, 2010, Ch. 11, pp. 235-251.

[5] J. Cheer, Active control of the acoustic environment in an automobile cabin, Ph.D. thesis, University of Southampton, Southampton, UK (2012).

[6] T. J. Sutton, S. J. Elliott, A. M. McDonald, T. J. Saunders, Active control of road noise inside vehicles, Noise Control Engineering Journal 42 (4) (1994) 137-146.

7] S. J. Elliott, I. M. Stothers, P. A. Nelson, A. M. McDonald, D. C. Quinn, T. J. Saunders, The active control of engine noise inside cars, in: INTER-NOISE and NOISE-CON Congress and Conference Proceedings, Vol. 1988, Institute of Noise Control Engineering, 1988, pp. 987-990.

[8] J. Cheer, S. J. Elliott, Multichannel control systems for the attenuation of interior road noise in vehicles, Mechanical Systems and Signal Processing 60 (2015) 753-769.

[9] R. Schirmacher, R. Kunkel, M. Burghardt, Active noise control for the 4.0 tfsi with cylinder on demand technology in audi's s-series, Tech. Rep. No. 2012-01-1533, SAE Technical Paper (2012).

360 [10] T. Inoue, A. Takahashi, H. Sano, M. Onishi, Y. Nakamura, Nv countermeasure technology for a cylinder-on-demand engine-development of active booming noise control system applying adaptive notch filter, Tech. Rep. No. 2004-01-0411, SAE Technical Paper (2004).

[11] H. Sano, T. Inoue, A. Takahashi, K. Terai, Y. Nakamura, Active control system for low-frequency road noise combined with an audio system, IEEE Transactions on speech and audio processing 9 (7) (2001) 755-763.

365 [12] K. Sakamoto, T. Inoue, Development of feedback-based active road noise control technology for noise in multiple narrowfrequency bands and integration with booming noise active noise control system, SAE International Journal of Passenger Cars-Mechanical Systems 8 (2015-01-0660) (2015) 1-7. 
[13] B. Rafaely, S. J. Elliott, J. Garcia-Bonito, Broadband performance of an active headrest, The journal of the Acoustical Society of America 106 (2) (1999) 787-793.

370 [14] M. Pawelczyk, Adaptive noise control algorithms for active headrest system, Control Engineering Practice 12 (9) (2004) 1101-1112.

[15] P. Booij, A. P. Berkhoff, Virtual sensors for local, three dimensional, broadband multiple-channel active noise control and the effects on the quiet zones, in: 24th International Conference on Noise and Vibration Engineering, ISMA 2010, Katholieke Universiteit Leuven, 2010, pp. 151-166.

375 [16] J. Garcia-Bonito, S. J. Elliott, C. C. Boucher, Generation of zones of quiet using a virtual microphone arrangement, The journal of the Acoustical Society of America 101 (6) (1997) 3498-3516.

[17] A. Roure, A. Albarrazin, The remote microphone technique for active noise control, in: INTER-NOISE and NOISE-CON Congress and Conference Proceedings, Vol. 1999, Institute of Noise Control Engineering, 1999, pp. $1233-1244$.

[18] C. D. Petersen, R. Fraanje, B. S. Cazzolato, A. C. Zander, C. H. Hansen, A kalman filter approach to virtual sensing for active noise control, Mechanical Systems and Signal Processing 22 (2) (2008) 490-508.

[19] S. J. Elliott, P. Joseph, A. J. Bullmore, P. A. Nelson, Active cancellation at a point in a pure tone diffuse sound field, Journal of sound and vibration 120 (1) (1988) 183-189.

[20] D. Moreau, B. Cazzolato, A. Zander, C. Petersen, A review of virtual sensing algorithms for active noise control, Algorithms 1 (2) (2008) 69-99.

385 [21] S. J. Elliott, M. Simon, J. Cheer, W. Jung, Head tracking for local active noise control, in: 12th Western Pacific Acoustics Conference, 2015.

[22] W. Jung, S. J. Elliott, J. Cheer, Combining the remote microphone technique with head-tracking for local active sound control, The Journal of the Acoustical Society of America 142 (1) (2017) 298-307.

[23] D. Treyer, S. Gaulocher, S. Germann, E. Curiger, Towards the implementation of the noise-cancelling office chair: Algorithms and practical aspects, in: 23rd Int. Congress on Sound \& Vibration Proceedings, 2016.

[24] W. Jung, S. J. Elliott, J. Cheer, Local active sound control using the remote microphone technique and head-tracking for tonal and broadband noise sources, in: 24th Int. Congress on Sound \& Vibration Proceedings, 2017.

[25] S. J. Elliott, Signal processing for active control, Academic press, London, 2000.

[26] S. J. Elliott, J. Cheer, Modeling local active sound control with remote sensors in spatially random pressure fields, The journal of the Acoustical Society of America 137 (4) (2015) 1936-1946.

[27] S. J. Elliott, T. J. Sutton, Performance of feedforward and feedback systems for active control, IEEE Transactions on Speech and Audio Processing 4 (3) (1996) 214-223. 